\title{
Hybrid chemical etching of femtosecond irradiated 3D structures in fused silica glass
}

\author{
Sara Lo Turco ${ }^{1,2}$, Roberto Osellame ${ }^{3}$, Roberta Ramponi ${ }^{1,2,3}$ and \\ Krishna Chaitanya Vishnubhatla ${ }^{{ }^{*}}$ \\ ${ }^{1}$ Center for Nano Science and Technology@PoliMi, Istituto Italiano di Tecnologia, Via Giovanni Pascoli, 70/3, 20133, Milan, Italy \\ ${ }^{2}$ Dipartimento di Fisica, Politecnico di Milano, Piazza Leonardo da Vinci 32, 20133, Milan, Italy \\ ${ }^{3}$ Istituto di Fotonica e Nanotecnologie - CNR, Piazza Leonardo da Vinci 32, 20133, Milan, Italy \\ krishna.vishunubhatla@iit.it
}

\begin{abstract}
We report on the fabrication of 3D micro-structures in fused silica glass using chemical etching along femtosecond laser irradiated zones. In particular, we exploited a novel approach combining two different etching agents in successive steps: the hydrofluoric acid, which provides fast volume removal, and potassium hydroxide, which exhibits high selectivity. We demonstrated that this hybrid approach enables a more easy prototyping and fabrication of complex geometries for microfluidic devices.
\end{abstract}

\section{Introduction}

Optofluidic incorporates the fields of optics and microfluidics onto a single platform, giving rise to miniaturized Lab on Chip systems. Femtosecond laser irradiation and chemical etching (FLICE) is a reliable technique for the fabrication of microfluidic elements owing to its inherent ability to realize buried structures [1]. FLICE consists of two steps:

1) irradiation of the sample with focused femtosecond laser pulses so as to inscribe the device path;

2) wet chemical etching to remove primarily the laser modified zones. The standard etchant used to perform etching of silica glass substrates is the hydrofluoric acid in aqueous solution (HF). The HF etching turns out to be isotropic in the space because also the un-irradiated silica is attacked, although at a much lower rate, thus the microchannels result with a conical shape since the terminations are exposed to the acid for a longer time. Concerning this peculiarity, a proper selection of the etching and writing parameters is required to get uniform channels as long as few millimeters [2-5]. To improve the aspect ratio, Kiyama and co-workers [6] suggested the use of potassium hydroxide (KOH) for the etching step. They reported the formation of microchannels as long as $9.2 \mathrm{~mm}$ with a uniform diameter of the order of $60 \mathrm{um}$. The main drawback of $\mathrm{KOH}$ is that the etching rates are smaller as compared to HF acid. Moreover total volume irradiation is necessary for removal of larger structures.

\section{Experimental methods}

The laser irradiation of the sample is carried out by the second harmonic $(515 \mathrm{~nm})$ of the femtosecond laser pulses from regeneratively amplified diode-pumped mode-locked Yb:KGW laser $(1030 \mathrm{~nm}, 500 \mathrm{kHz}, 280 \mathrm{fs})$. The laser beam is focused inside a $1 \mathrm{~mm}$-thick fused silica substrate using the following microscope objectives: 50X (NA 0.42); 100X immersion-oil (NA 1.25). The pulse energy is set at $300 \mathrm{~nJ}$, for NA 0.42 , and $25 \mathrm{~nJ}$ for NA 1.25 , in order to be in the fluence range facilitating the formation of nano-gratings [4]. The sample is mounted on a 3 axis translation stage. Transversal writing geometry $(1 \mathrm{~mm} / \mathrm{s})$ is adopted and the laser writing direction is perpendicular to the beam polarization. The etching with $\mathrm{HF}$ is performed with a $20 \%$ concentration aqueous solution, placed inside an ultrasonic bath at $35^{\circ} \mathrm{C}$. For etching with $\mathrm{KOH}$, a 10 molar solution in water at $90^{\circ} \mathrm{C}$ along with a magnetic stirrer was used.

\section{Results and Discussion}

In the following examples we demonstrate where $\mathrm{HF}$ acid is to be used and where $\mathrm{KOH}$ performs better, and overall how their combination improves the etching process and allows the fabrication of more complex optofluidic devices..

3.1 Microchannels with vertical access holes

With the aim of realizing microchannels with uniform cylindrical cross section and vertical access holes, laser irradiation was performed in 2 fused silica chips in the form of a single line at a depth of $400 \mu \mathrm{m}$ with a length of $2 \mathrm{~mm}$, using the 50X 0.42 N.A. The first chip was etched in $20 \% \mathrm{HF}$ in ultrasonic bath at $35^{\circ} \mathrm{C}$. The second was etched in $\mathrm{KOH}$ solution. The etching starts from the surface of the sample forming the two access holes first, and then the channel is etched from both the sides. The results are reported in figure 1 . As expected the channel etched in high concentration of HF showed a significant tapering $(10 \mu \mathrm{m}$ at the center as compared to $65 \mu \mathrm{m})$ but it took only 2 hours for the channel to be completely etched. On the contrary, the tapering effect is negligible when the etching is performed with $\mathrm{KOH}$, Fig. $1 \mathrm{~b}$, where the top view shows that after 7 hours the microchannel manifests a uniform diameter of $10 \mu \mathrm{m}$. The latter channel was further etched in HF for 30 minutes to increase the diameter. It may be noted that once there is a through channel for the flow of HF acid, there would be no further tapering, Fig. 1c, and the result is a uniform channel with diameter of $45 \mu \mathrm{m}$. 

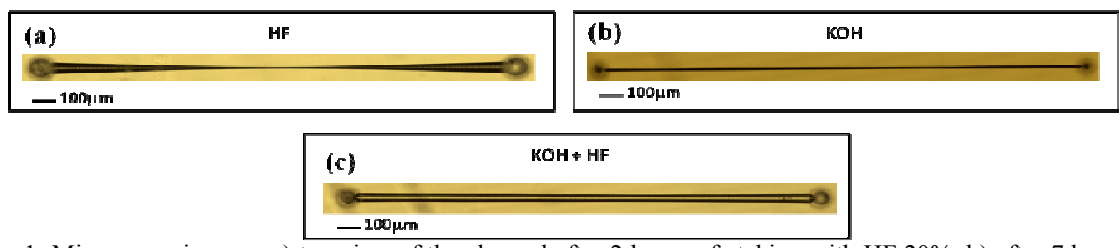

Figure1: Microscope images: a) top view of the channel after 2 hours of etching with HF $20 \%$; b) after 7 hours of etching with $\mathrm{KOH}$; c) channel realized after 7 hours of etching with $\mathrm{KOH}$ further etched with $\mathrm{HF} 20 \%$ for 30 min.

Thus it is evident that $\mathrm{KOH}$ is effective in etching small features, with respect to HF etching. On the other hand using only $\mathrm{KOH}$ for etching large volumes of fused silica glass would imply either long etching time or the need for laser irradiation of the entire volume. Hence the choice of etchant has to be judicious depending on the device. In the following section we demonstrate an example of application where HF is used to remove larger volumes, while $\mathrm{KOH}$ is used for small zones where extreme selectivity is required.

3.2 Micro-filter

One of the most important components of a microfluidic platform are microfilters suitable to separate different species on a micrometer size scale. In this device the filter is directly encapsulated inside a square channel $80 \mu \mathrm{m} x 80 \mu \mathrm{m}$ wide, $2.25 \mathrm{~mm}$ long: the filtration stage is composed by a grid $(15 \times 15)$ of microchannels placed at the centre of the square channel; the microchannels have a maximum diameter of $2.05 \mu \mathrm{m}$, a total length of $100 \mu \mathrm{m}$ and are spaced apart by $5 \mu \mathrm{m}$. The whole structure is written with the immersion oil $1.25 \mathrm{NA}$ objective at a central depth of $160 \mu \mathrm{m}$.

As it is clear from Fig. 2a, after the writing step three different zones can be distinguished: A) where only the borders of the structure are written; B) closer to the filter, this is a parallelepiped of $80 \mu \mathrm{m} \times 80 \mu \mathrm{m}$ completely written in the volume with $1 \mu \mathrm{m}$-spaced lines; $\mathrm{C}$ ) the filter grid itself. Zone $\mathrm{A}$ is the longest part of the channel and has to be etched with HF; zone B can be etched either with $\mathrm{KOH}$ or HF and it is a sort of 'safety zone' to avoid that the final step of $\mathrm{HF}$ could attack the filter; finally zone $\mathrm{C}$ has to be etched only with $\mathrm{KOH}$ in order to avoid merging of the filtermicrochannels.

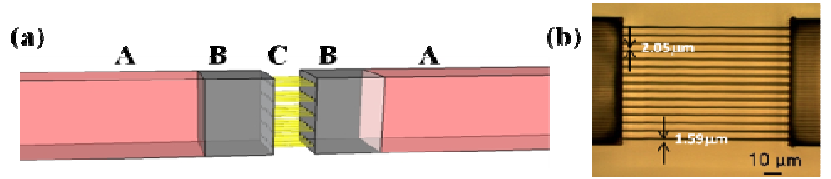

Figure 2: (a) Schematic diagram of the device in $3 \mathrm{D}$ with difference zones, where $\mathrm{HF}$ or $\mathrm{KOH}$ is used for etching (see the text); (b) Microscope image of the filter structure after etching.

(a)

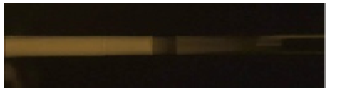

(b)

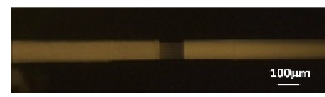

(i)

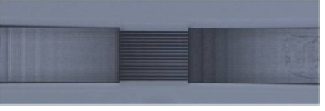

(d)

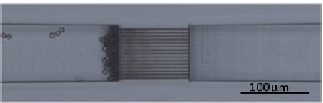

Figure 3: Microscope images of $(\mathrm{a}, \mathrm{b})$ fluorescence from Rhodamine $6 \mathrm{G}$ dye flowing from one arm (left side) to the other arm (right side); (c) Device filled with water, (d) beads $7 \mu \mathrm{m}$ in size stopped at the filter surface.

Once the filter is etched out, a solution of Rhodamine $6 \mathrm{G} 40 \mu \mathrm{Mol}$ is flowed through the channel and the fluorescence is imaged. Fig. 3a-b clearly depict the smooth flow from one arm of the channel to the other through the filtering structure indicating that indeed the two arms are connected by the filtering pores. To test the filtering operation, water solutions with polystyrene beads inside $(7 \mu \mathrm{m}$ in size) were flowed through the device. Fig. 3c-d display the time evolution of the flow. Water flows through to the second arm of the channel, while the micro-beads are stopped at the filter entrance.

\section{Conclusions}

In the current work we showed a novel hybrid chemical etching of femtosecond laser irradiated structures. Using this engineered approach we demonstrated the fabrication of a microfilter embedded in fused silica glass, to separate different species on a micrometer size scale. With the new degree of freedom achieved by the combination of fast etching rate of $\mathrm{HF}$ with the high aspect ratios obtained by $\mathrm{KOH}$ etching, it will be possible to realize complex 3D structures which could not be fabricated using either HF or KOH solutions individually.

\section{References}

[1] R. Osellame, H. J. W. M. Hoekstra, G. Cerullo, and M. Pollnau, "Femtosecond laser microstructuring: an enabling tool for optofluidic lab-onchips," Laser Photonics Rev. 5, 442-463 (2011).

[2] A. Marcinkevicius, S. Juodkazis, M. Watanabe, M. Miwa, S. Matsuo, H. Misawa, and J. Nishii, "Femtosecond laser-assisted three-dimensional microfabrication in silica," Opt. Lett. 26, 277 (2001).

[3] Y. Bellouard, A. Said, M. Dugan, and P. Bado, "Fabrication of high-aspect ratio, micro-fluidic channels and tunnels using femtosecond laser pulses and chemical etching," Opt. Express 12, 2120-2129 (2004).

[4] C. Hnatovsky, R. Taylor, E. Simova, V. Bhardwaj, D. Rayner, and P. Corkum, "Polarization-selective etching in femtosecond laser-assisted microfluidic channel fabrication in fused silica," Opt. Lett. 30, 1867 (2005).

[5] V. Maselli, R. Osellame, G. Cerullo, R. Ramponi, and P. Laporta, "Fabrication of long microchannels with circular cross section using astigmatically shaped femtosecond laser pulses and chemical etching," Appl. Phys. Lett. 88, 191107 (2006).

[6] S. Kiyama, S. Matsuo, S. Hashimoto, and Y. Morihira, "Examination of Etching Agent and Etching Mechanism on Femotosecond Laser Microfabrication of Channels Inside Vitreous Silica Substrates," J. Phys. Chem. C 113, 11560 (2009). 